\title{
ON THE PERIOD-TWO-PROPERTY OF THE MAJORITY OPERATOR IN INFINITE GRAPHS
}

\author{
GADI MORAN
}

\begin{abstract}
A self-mapping $M: X \rightarrow X$ of a nonempty set $X$ has the PeriodTwo-Property (p2p) if $M^{2} x=x$ holds for every $M$-periodic point $x \in X$. Let $X$ be the set of all $\{0,1\}$-labelings $x: V \rightarrow\{0,1\}$ of the set of vertices $V$ of a locally finite connected graph $G$. For $x \in X$ let $M x \in X$ label $v \in V$ by the majority bit that $x$ applies to its neighbors, retaining $v$ 's $x$-label in case of a tie. We show that $M$ has the $\mathrm{p} 2 \mathrm{p}$ if there is a finite bound on the degrees in $G$ and $\frac{1}{n} \log b_{n} \rightarrow 0$, where $b_{n}$ is the number of $v \in V$ at a distance at most $n$ from a fixed vertex $v_{0} \in V$.
\end{abstract}

\section{INTRODUCTION}

Let us say that a graph $G$ has the period-two-property if the majority action on the set of 0-1 labelings of its vertices (see abstract or (1.1)) has the p2p. The $\mathrm{p} 2 \mathrm{p}$ for finite graphs was observed in the late 1970s, when it appeared as a special instance in several interesting classes of discrete-time dynamical systems with the $\mathrm{p} 2 \mathrm{p}$ ([GO], [PS], [PT]; see [GM] for an extensive survey and further references). The need to establish the $\mathrm{p} 2 \mathrm{p}$ for some infinite graphs arose in our recent treatment of the $r$-majority operator $M_{r}$ on two-way-infinite 0 1-sequences. $M_{r}$ replaces simultaneously each bit of such a sequence by the majority bit of the $(2 r+1)$-interval it centers [M1], [M2]. It follows from Theorem 1 , that the $r$-majority operator indeed has the $\mathrm{p} 2 \mathrm{p}$. Moreover, it follows that if the Euclidean distance $\rho_{k}(u, v)$ between any two members $u, v$ of a subset $V$ of the $k$-dimensional Euclidean space exceeds some fixed positive distance $h$ and if a graph $G$ is formed by joining $u$ and $v$ in $V$ only if $0<\rho_{k}(u, v) \leq r$ for some fixed positive number $r$, then the majority operator on this graph $G$ has the p2p (Corollary 1.7). In particular, the p2p holds when $V$ is the set of "sites" in some infinite lattice in a Euclidean space, each site is occupied with a "spin", say + or - , and the spins undergo simultaneous change at discrete time steps, where the spin at site $v \in V$ at time $t+1$ is + or - according to the majority of spins in the ball of radius $r$ centered at $v$ at time $t$ (changing its spin in the case of a tie). Thus, if any given distribution of spins at $t=0$ ever reappears at some future time, it reappears at $t=2$.

Received by the editors February 11, 1993 and, in revised form, May 16, 1994.

1991 Mathematics Subject Classification. Primary 68R10, 49A21, 49D50; Secondary 68Q80, 68Q90, 90A08, 94B30.

Key words and phrases. Period-two, majority rule, cellular automata, infinite graphs. 
As the p2p holds for a graph $G$ iff it holds for each of its connected components, we assume in the sequel that $G$ is connected. Theorem 1 guarantees the p2p for $G$ if two conditions are satisfied, namely

BD: vertex degrees have a finite bound,

and

SEG: $G$ has subexponential growth (see (1.8) and Definition 1.5, §1). As BD and SEG trivally hold for finite graphs, Theorem 1 is an extension of the familiar $\mathrm{p} 2 \mathrm{p}$ for finite graphs. In a sense, this extension is sharp: Examples 1 and 2 in $\S 1$ present respectively graphs without BD but with SEG and with BD without SEG, that lack the p2p (Remark 1.6).

There is, in fact, a quantitative trade-off between the restrictions expressed by the conditions BD and SEG. A numerical parameter $g(G)$ defined for a connected locally finite graph $G$-the growth of $G$ (Definition 1.5)-satisfies $1 \leq g(G) \leq d(G)-1$ whenever $d(G)>1$ is the maximum degree of the graph $G$ satisfying BD. The SEG simply means that $g(G)=1$. Theorem 2-which implies Theorem 1 -tells us that in fact, if $g(G)<1+\frac{2}{d}$, where $d$ is the largest even integer satisfying $d<d(G)$, then $G$ has the p2p. This result is sharp: in Example 2-where the p2p fails-we have $g(G)=1+\frac{2}{d}$.

In $\S 1$ we offer precise definitions and formulation of the results.

In $\S 2$ we give a necessary and sufficient condition for a self-mapping $M$ of an abstract set $X$ to have the p2p, namely, the existence of a p2p form for $M$ (Theorem 2.0). Let us briefly explain this notion.

We say that $M$ solves a binary form $b: X \times X \rightarrow P$ of $X$ into a partial order $P$ if for each $x \in X \quad M x$ maximizes $b(x, y)$ over all $y \in X$. The form $b$ is called a $p 2 p$ form for $M$ in $P$ if $b$ is symmetric, $M$ solves $b$, and moreover, $M x$ is the only solution in $X$ to the maximization problem "maximize $b(x, y)$ over $y \in X$ ".

Theorem 3.0 in $\S 3$ provides convenient general sufficient conditions for "locally" defined operators on networks to have the $\mathrm{p} 2 \mathrm{p}$. Its specialization Theorem 3.1 puts these conditions in a form useful for proving Theorem 2 in $\S 4$.

The conditions BD and SEG of Theorem 1 suffice to ensure the $p 2 p$ for a wide range of operators other than $M$. As a rule, each such operation admits some quantitative condition relating $g(G)$ to $d(G)$ (as Theorem 2 does in $M$ 's case) securing its $\mathrm{p} 2 \mathrm{p}$, the way Theorem 2 implies Theorem 1 . The quantitative condition, however, is sensitive to the particular operator under consideration and follows from an analysis of the "local" rule defining it. The systematic extension of known $\mathrm{p} 2 \mathrm{p}$ theorems for operators on finite graphs-or more generally, on finite networks-to the infinite by spelling out the relevant quantitative condition is beyond the scope of this paper and will hopefully be carried out in the future. Here we shall illustrate the situation by establishing the $\mathrm{p} 2 \mathrm{p}$ for one more operator-the majority action $M^{(k)}$ on $k$-colored graphs. $M^{(k)}$ acts on functions $x: V \rightarrow\{0, \ldots, k-1\}$-where the tie-breaking rule is "least-best": $M^{(k)} x(v)$ is the smallest $c \in\{0, \ldots, k-1\}$ which is taken by $x$ most often in the neighbourhood of the vertex $v$ of a locally finite graph $G$ (see (1.27)). The $\mathrm{p} 2 \mathrm{p}$ for this operation on finite graphs is proved in [PS]. Theorem 3.2-a slight generalization of Theorem 3.1 and another immediate corollary of Theorem 3.0-is suitable for establishing Theorem 3, which states that $M^{(k)}$ has the $\mathrm{p} 2 \mathrm{p}$ in arbitrary graphs with BD and SEG. Theorem 3.2 is 
used to prove Theorem 4-the quantitative result yielding Theorem 3-which states that $M^{(k)}$ acting on a connected graph $G$ with maximum finite degree $d>1$ has the $\mathrm{p} 2 \mathrm{p}$ if $g(G)<\left(1+\frac{2}{d-1}\right)^{1 / k}$.

\section{NOTATION AND RESULtS}

We let $\mathbb{N}, \mathbb{N}_{0}$, and $\mathbb{Z}$ denote the sets of positive, nonnegative, and all integers respectively. For $n \in \mathbb{N}, \mathbb{Z}_{n}:=\{0,1, \ldots, n-1\} . \mathbb{R}$ denotes the set of real numbers. $|A|$ denotes the cardinality of a set $A . A \times B$ and ${ }^{B} A$ denote the cartesian product of $A$ by $B$ and the set of all functions, or mappings, from $B$ into $A$; i.e.

$$
\begin{aligned}
A \times B: & =\{(a, b) \mid a \in A, b \in B\}, \\
{ }^{B} A: & =\{x \mid x: B \rightarrow A\} .
\end{aligned}
$$

A graph $G=(V, N)$ consists of a nonempty set $V$ of vertices and an irreflexive symmetric binary relation $N \subseteq V \times V$. Writing $u N v$ for $(u, v) \in$ $N$, this means

(N1) $\neg v N v \quad(v \in V)$,

(N2) $u N v \Rightarrow v N u \quad(u, v \in V)$.

We say that $u$ is a neighbour of $v$ (in $G$ ) if $u N v$ and accordingly call the set

$$
N_{v}:=\{u \in V: u N v\}
$$

the neighbourhood of $v$ in $G$. Notice that by (N1), $v$ does not belong to $N_{V}$.

The cardinal number $d_{G}(v):=\left|N_{v}\right|$ is called the degree of $v$.

Fix a graph $G . G$ is called locally finite if $d_{G}(v)$ is finite for every $v \in V$. $G$ is called finite if $V$ is finite.

Let $C$ be a nonempty set. A function $x: V \rightarrow C$ is referred to as a $C$ coloring, a $C$-labeling or a $C$-Configuration of $V$ (or of $G$ ). We let $X:={ }^{V} C$ denote the set of all $C$-configurations; the set $C$ will be clear from the context (or irrelevant).

Given a $C$-configuration $x: V \rightarrow C$ and $v \in V, N_{v}$ is partitioned by $x$ into (possibly empty) color-subsets $N_{v}(x, c), c \in C$, of cardinalities $d_{v}(x, c)$. That is,

$$
\begin{aligned}
N_{v}(x, c) & :=\left\{u \in N_{v}: x(u)=c\right\}, \\
d_{v}(x, c) & :=\left|N_{v}(x, c)\right| \quad(v \in V, x \in X, c \in C) .
\end{aligned}
$$

Now assume that $G$ is locally finite, $C=\mathbb{Z}_{2}=\{0,1\}$, and let $x: V \rightarrow \mathbb{Z}_{2}$ be a $\mathbb{Z}_{2}$-configuration. Then a $\mathbb{Z}_{2}$-configuration $M x$ is defined by

$$
\begin{array}{ll}
M x(v):=c & \text { if } d_{G}(v)<2 d_{v}(x, c), \\
M x(v):=x(v) & \text { if } d_{G}(v)=2 d_{v}(x, c) .
\end{array}
$$

The mapping $M: X \rightarrow X$ so defined is called the majority operation on the set of $\mathbb{Z}_{2}$-configurations of $G$, or, briefly, the majority operation on $G$. Notice that (1.1) defines $M$ properly whenever the set $C$ of colors contains no more than two elements.

For the arbitrary self-mapping $M: X \rightarrow X$ of an arbitrary set $X$ and any $n \in \mathbb{N}_{0}, M^{n}: X \rightarrow X$ is defined inductively as usual by $M^{0} x=x, M^{n+1} x=$ 
$M\left(M^{n} x\right) \quad(x \in X)$. We say that $M$ has the period-two-property (p2p) if it satisfes

$$
\forall x \in X \forall n \in \mathbb{N}\left[M^{n} x=x \Rightarrow M^{2} x=x\right] .
$$

This paper generalizes:

Theorem 1.0 [GO]. Let $G$ be a finite graph. Then the majority operator defined by (1.1) acting on $X=V^{V} \mathbb{Z}_{2}$ has the p2p.

Now we present two basic examples of (infinite, locally finite) graphs indicating that the word "finite" cannot be dropped in Theorem 1.0 and suggesting its generalization, Theorem 1 below. First some more notation.

Let $G=(V, N)$ be a locally finite graph, and let the sequence of sets $\mathbf{V}:=$ $\left(V_{n}\right)_{n \in \mathbb{N}_{0}}$ satisfy $V_{n} \neq \varnothing, V_{n} \cap V_{m}=\varnothing$ for $n \neq m$, and $V=\bigcup_{n \in \mathbb{N}_{0}} V_{n}$. Let $C$ be a set (of "colours"), and for $\delta: \mathbb{N}_{0} \rightarrow C$ define $x_{\delta}: V \rightarrow C$ by

$$
\forall n \in \mathbb{N}_{0} \forall v \in V_{n}\left[x_{\delta}(v)=\delta(n)\right] .
$$

Now let $C=\mathbb{Z}_{2}$. We say that $V$ is a shift system for $M$ if for every $\delta: \mathbb{N}_{0} \rightarrow C$

$$
\forall n \in \mathbb{N}_{0} \forall v \in V_{n}\left[M x_{\delta}(v)=\delta(n+1)\right] .
$$

For $\delta: \mathbb{N}_{0} \rightarrow C$ define $S \delta: \mathbb{N}_{0} \rightarrow C$ by

$$
S \delta(n):=\delta(n+1) \text {. }
$$

Thus $\mathbf{V}$ is a shift system for $G$ iff for every $\delta$

$$
M x_{\delta}=x_{S \delta}
$$

i.e., if $x=x_{\delta}$, then $M x$ assigns to $V_{n}$ the color that $x$ assigns to $V_{n+1}$.

Assume that $\mathbf{V}$ is a shift system for $G$. Then $G$ violently violates the p2p. Indeed for any $\delta: \mathbb{N}_{0} \rightarrow \mathbb{Z}_{2}, n, k \in \mathbb{N}_{0}$, and any $v \in V_{n}$ we have $M^{k} x_{\delta}(v)=$ $\delta(n+k)$. Hence, if $r \in \mathbb{N}$ and $\delta: \mathbb{N}_{0} \rightarrow \mathbb{Z}_{2}$ is defined by $\delta(n)=1$ iff $n$ is divisible by $r$, then the $\mathbb{Z}_{2}$-configuration $x:=x_{\delta}$ satisfies $M^{r} x=x$ but $M^{s} x \neq x$ for $s=1, \ldots, r-1$ and so (1.2) fails for $x$ if $r>2$.

Example 1. Let $\mathbf{V}=\left(V_{n}\right)_{n \in \mathbb{N}_{0}}$ be a sequence of disjoint finite sets $V_{n}$ satisfying $\left|V_{n}\right|=\left\lfloor\frac{n+2}{2}\right\rfloor$, and $V:=\bigcup_{n \in \mathbb{N}_{0}} V_{n}$. Define a graph $G_{1}=(V, N)$ whose set of vertices is $V$ and with the following neighbourhood relation $N$. Let $u, v \in V$ and let $m, n \in \mathbb{N}_{0}$ satisfy $u \in V_{n}, v \in V_{m}$. Then

$$
u N v: \Leftrightarrow|n-m|=1 \text {. }
$$

Now consider $v \in V_{n}$ for some $n \in \mathbb{N}$. Then $N_{v}=V_{n-1} \cup V_{n+1}$. For $\delta: \mathbb{N}_{0} \rightarrow$ $\mathbb{Z}_{2}$ and $c=\delta(n+1)$ we have by $(1.3) N_{v}\left(x_{\delta}, c\right) \supseteq V_{n+1}$, so $d_{v}\left(x_{\delta}, c\right) \geq$ $\left|V_{n+1}\right|>\frac{1}{2} d_{G}(v)$. Hence, by $(1.1)\left(M x_{\delta}\right)(v)=c$, i.e., $\left(M x_{\delta}\right)(v)=\delta(n+1)$. Thus, $V$ is a shift system for $G_{1}$.

Remark 1.1. Let $|C| \geq 2$, and let $X$ denote the set of $C$-configurations of the graph $G_{1}$. Let further $\widetilde{M}: X \rightarrow X$ be any mapping satisfying

$$
(\widetilde{M} x)(v)=c \quad \text { if } \forall c^{\prime} \in C\left[c^{\prime} \neq c \Rightarrow d_{v}\left(x, c^{\prime}\right)<d_{v}(x, c)\right] .
$$

For any $\delta: \mathbb{N}_{0} \rightarrow C$ define $x_{\delta}: V \rightarrow C$ by (1.3). Then (1.5) still holds. In particular, $\widetilde{M}$ does not have the p2p. 
Remark 1.2. We have $d_{G}(v)=1$ for $v \in V_{0}$; and for $v \in V_{n}, n>0$,

$$
d_{G}(v)=\left|V_{n-1}\right|+\left|V_{n+1}\right|=\left\lfloor\frac{n+1}{2}\right\rfloor+\left\lfloor\frac{n+3}{2}\right\rfloor,
$$

i.e.

$d_{G}(v)=n+1$ or $n+2$ accordingly as $n$ is even or odd.

$G_{1}$ fails to have the $\mathrm{p} 2 \mathrm{p}$; and by Remark 1.2 , the degree function $d_{G}(v)$ has no finite bound either.

Let us define, for an arbitrary graph $G$, the cardinal invariant $d(G)$ by

$$
d(G):=\sup \left\{d_{G}(v): v \in V\right\} .^{1}
$$

Thus, although $G_{1}$ is locally finite, $d\left(G_{1}\right)=\aleph_{0}$.

We say that a graph $G$ has $B D$ (bounded degree) if $G$ satisfies:

$$
d(G)<\aleph_{0} \text {. }
$$

Let $k \in \mathbb{N}, k>1$. The full $k$-tree $T_{k}$ is an example of a graph satisfying (1.8) $\left(d\left(T_{k}\right)=k+1\right)$ yet having a shift system. By definition, $T_{k}=\dot{U}_{n \in \mathbf{N}_{0}} V_{n}$ where $V_{n}={ }^{\left(\mathbb{Z}_{n}\right)} \mathbb{Z}_{k}$ is the set of all sequences $\left(i_{0}, \ldots, i_{n-1}\right)$ of length $n$ with $i_{j} \in \mathbb{Z}_{k}, j=0, \ldots, n-1$. Let $u \prec v$ stand for " $u$ is an initial segment of $v$ " for $u, v \in T_{k}$; then the neighborhood relation $N$ in $T_{k}$ is given for $u \in V_{n}$, $v \in V_{m}$ by

$$
u N v: \Leftrightarrow|n-m|=1 \&(u \prec v \text { or } v \prec u) \text {. }
$$

Obviously, $\mathbf{V}=\left(\dot{V}_{n}\right)_{n \in \mathbb{N}_{0}}$ is a shift system for $T_{k}$, and $d\left(T_{k}\right)=k+1$. Notice, however, that here $\left|V_{n}\right|=k^{n}$. Anticipating the condition SEG, we present next an example of $G$ satisfying (1.8) with a slow-growing shift system.

Example 2. Let $k \in \mathbb{N}, k>1$. Define by induction $l_{n}, q_{n}, r_{n}, \varepsilon_{n} \in \mathbb{N}_{0}$ for $n \in \mathbb{N}_{0}$ by

$$
\begin{aligned}
& l_{0}=1 ; \\
& l_{n}=q_{n} \cdot k+r_{n}, \quad 0 \leq r_{n}<k ; \\
& \varepsilon_{n}=0 \quad \text { if } r_{n}=0, \quad \varepsilon_{n}=1 \quad \text { if } r_{n}>0 \\
& l_{n+1}=\left(q_{n}+\varepsilon_{n}\right)(k+1) .
\end{aligned}
$$

We define the graph $G_{2}^{(k)}$ as follows:

Let $\mathbf{V}=\left(V_{n}\right)_{n \in \mathbb{N}_{0}}$ be a sequence of mutually disjoint finite sets satisfying $\left|V_{n}\right|=l_{n}\left(n \in \mathbb{N}_{0}\right)$. For each $n$ let $P_{n}$ be a partition of $V_{n}$ into $q_{n}+\varepsilon_{n}$ nonempty sets, all but possibly one of cardinality $k$, where the exceptional set exists iff $\varepsilon_{n}=1$ and then it is of cardinality $r_{n}$. Let $Q_{n+1}$ be a partition of $V_{n+1}$ into $q_{n}+\varepsilon_{n}$ sets of cardinality $k+1$, and let $f_{n+1}: Q_{n+1} \rightarrow P_{n}$ be a bijection of $Q_{n+1}$ onto $P_{n}$. For $u \in V_{n}$ let $\widetilde{P}_{n}(u)$ denote the unique set $V^{\prime} \subseteq V_{n}, V^{\prime} \in P_{n}$, satisfying $u \in V^{\prime}$; and for $v \in V_{n+1}$ let $\widetilde{Q}_{n+1}(v)$ denote the unique set $V^{\prime \prime} \subseteq V_{n+1}, V^{\prime \prime} \in Q_{n+1}$, satisfying $v \in V^{\prime \prime}$. We first define an irreflexive antisymmetric relation $N^{\prime}$ on the set $V:=\bigcup_{n \in \mathbb{N}_{0}} V_{n}$ by

$$
u N^{\prime} v: \Leftrightarrow \exists n \in \mathbb{N}_{0}\left[u \in f_{n+1}\left(\widetilde{Q}_{n+1}(v)\right)\right] \text {. }
$$

1 If $A$ is a set of cardinal numbers, $\sup A$ is the least cardinal $m$ satisfying $a \leq m$ for all $a \in A$. For (1.7) to make sense in general the axiom of choice $A C$ is required, but if $V$ is a well-ordered set-in particular, if $V$ is countable- $A C$ is not needed. 
The graph $G_{2}^{(k)}=(V, N)$ with the set of vertices $V$ is obtained by defining the neighbourhood relation $N$ by

$$
u N v: \Leftrightarrow u N^{\prime} v \text { or } v N^{\prime} u,
$$

where $N^{\prime}$ is given by (1.10).

One readily checks that if $v_{0}$ is the only member of $V_{0}$, then

$$
N_{v_{0}}=V_{1}=f_{1}^{-1}\left(V_{0}\right) \text {. }
$$

Let $n \in \mathbb{N}$ and let $v \in V_{n}$. Then we have

$$
N_{v}=f_{n}\left(\widetilde{Q}_{n}(v)\right) \cup f_{n+1}^{-1}\left(\widetilde{P}_{n}(v)\right) .
$$

As $f_{n}\left(\widetilde{Q}_{n}(v)\right) \subseteq V_{n-1}, f_{n+1}^{-1}\left(\widetilde{P}_{n}(v)\right) \subseteq V_{n+1}$ and

$$
\left|f_{n}\left(\widetilde{Q}_{n}(v)\right)\right| \leq k, \quad\left|f_{n+1}^{-1}\left(\widetilde{P}_{n}(v)\right)\right|=k+1
$$

we see that $V$ is indeed a shift system for $G_{2}^{(k)}$ and that $d_{G}(v) \leq 2 k+1$. As $d_{G}(v)=2 k+1$ whenever $\left|f_{n}\left(\widetilde{Q}_{n}(v)\right)\right|=k$ we have

$$
d\left(G_{2}^{(k)}\right)=2 k+1 \text {. }
$$

We show that the sequences $\left(q_{n}\right)_{n \in \mathbb{N}_{0}},\left(l_{n}\right)_{n \in \mathbb{N}_{0}}$ are increasing sequences of integers. Moreover, setting

$$
g:=1+\frac{1}{k}
$$

both sequences are approximately geometric sequences with quotient $g$.

Indeed by (1.9) we have $l_{0}=1, q_{0}=0, l_{1}=k+1, q_{1}=1$ (as $\left.k>1\right)$, and also

$$
\begin{array}{ll}
l_{n}=n(k+1), & q_{n}=r_{n}=n(n=1, \ldots, k-1), \\
l_{k}=k(k+1), & q_{k}=k+1 .
\end{array}
$$

Assume $n \geq k$ and $q_{n}>n$. Then by (1.9)

$l_{n}=q_{n} k+r_{n}<q_{n} k+k<q_{n} k+q_{n}=q_{n}(k+1) \leq l_{n+1}<q_{n+1} k+k \quad(n \geq k)$.

Hence

$$
l_{n}<l_{n+1}, \quad q_{n}<q_{n+1} \quad\left(n \in \mathbb{N}_{0}\right) .
$$

Dividing through $q_{n} k$ the inequalities

$$
q_{n}(k+1)<q_{n+1} k+k, \quad q_{n+1} k \leq\left(q_{n}+1\right)(k+1)
$$

we obtain, by (1.13),

$$
g-\frac{1}{q_{n}}<\frac{q_{n+1}}{q_{n}} \leq\left(1+\frac{1}{q_{n}}\right) g
$$

so that by (1.15) and (1.13) we have $\lim _{n \rightarrow \infty} \frac{q_{n+1}}{q_{n}}=1+\frac{1}{k}$.

As by $(1.9) \frac{q_{n+1}}{q_{n}}\left(1-\frac{1}{q_{n}+1}\right)=\frac{q_{n+1}}{q_{n}+1}<\frac{l_{n+1}}{l_{n}}<\frac{q_{n+1}+1}{q_{n}}=\frac{q_{n+1}}{q_{n}}+\frac{1}{q_{n}}$, we also have by (1.15) $\lim _{n \rightarrow \infty} \frac{l_{n+1}}{l_{n}}=1+\frac{1}{k}$. It thus follows that

$$
\lim _{n \rightarrow \infty} l_{n}^{1 / n}=1+\frac{1}{k} \text {. }
$$


Hence, putting

$$
b_{n}=\sum_{0 \leq j \leq n} l_{j}
$$

we have (see Proposition A, Appendix)

$$
\lim _{n \rightarrow \infty} b_{n}^{1 / n}=1+\frac{1}{k}
$$

Remark 1.3. Remark 1.1 holds also for Example 2: If $C$ is any set satisfying $|C| \geq 2$ and we let $X:={ }^{V} C$ and $\widetilde{M}: X \rightarrow X$ satisfies (1.6), then (1.5) holds for every $\delta: \mathbb{N}_{0} \rightarrow C$. In particular, $\widetilde{M}$ does not have the p2p.

Now we define precisely the condition SEG (subexponential-growth). Let $G=(V, N)$ be a graph, $u, v \in V, n \in \mathbb{N}_{0}$. A trail of length $n$ from $u$ to $v$ (in $G$ ) is a sequence $\left(v_{0}, \ldots, v_{n}\right)$ of length $n+1$ satisfying $v_{0}=u, v_{n}=v$, and $v_{i} N v_{i+1}$ for $i \in \mathbb{Z}_{n}$. The distance $\rho(u, v)$ between $u$ and $v$ (in $G$ ) is by definition the smallest $n$ for which a trail of length $n$ from $u$ to $v$ exists. (It is undefined if no trail from $u$ to $v$ exists.) $G$ is connected if for any $u, v \in V$ a trail from $u$ to $v$ exists. When $G$ is connected, $\rho$ is a metric on $V$, taking values in $\mathbb{N}_{0}$.

For any $v \in V$ and $n \in \mathbb{N}_{0}$ we let $S(v, n)(B(v, n))$ denote the sphere (the closed ball) of radius $n$ centered at $v$ and let $s(v, n)(b(v, n))$ denote its cardinality. That is,

$$
\begin{array}{ll}
S(v, n):=\{u \in V: \rho(v, u)=n\}, & B(v, n):=\{u \in V: \rho(v, u) \leq n\}, \\
s(v, n):=|S(v, n)|, & b(v, n):=|B(v, n)| .
\end{array}
$$

As $S(v, 1)=N_{v}$ we have $s(v, 1)=d_{G}(v)$. Also, for all $n \in \mathbb{N}_{0}$ :

$$
\begin{aligned}
B(v, n) & =\bigcup_{0 \leq k \leq n} S(v, k), \quad b(v, n)=\sum_{0 \leq k \leq n} s(v, k) \\
\rho(v, w)=k \Rightarrow B(v, n) \subseteq B(w, n+k) \Rightarrow b(v, n) \leq b(w, n+k) & \left(v, w \in V, n, k \in \mathbb{N}_{0}\right) .
\end{aligned}
$$

Obviously $S(v, 0)=\{v\}, S(v, n+1) \subseteq \bigcup_{w \in S(v, n)} S(w, 1)$; and for $n>0$ and $w \in S(v, n), S(w, 1) \cap S(v, n-1) \neq \varnothing$. Thus, $B(v, n)$ is a finite set when $G$ is locally finite.

Now assume that $G$ has BD, i.e., (1.8) holds. Let $d=d(G)$ and let $v \in V$. Then $s(v, 0)=1, s(v, 1) \leq d$, and for $n \geq 1 s(v, n) \leq(d-1) s(v, n-1)$. Thus, by (1.20), for $d>2$

$$
b(v, n) \leq 1+d \frac{(d-1)^{n}-1}{d-2} \quad(d=d(G)>2, n \geq 0) .
$$

Equality in (1.22) holds if $d=k+1, G=T_{k}$, the full $k$-tree, and $v$ is the root of $T_{k}$. By (1.22):

$$
1 \leq b(v, n) \leq 3(d-1)^{n} \quad(d=d(G)>2, n \geq 0) .
$$

Define the growth $g(v, G) \in \mathbb{R} \cup\{\infty\}$ of $G$ at $v$ for any vertex $v$ of a locally finite graph $G$ by

$$
g(v, G):=\limsup _{n \in \mathbf{N}}(b(v, n))^{1 / n} .
$$


If $d(G)=d, 2<d \in \mathbb{N}$, then by (1.23) $1 \leq g(v, G) \leq d-1$.

We notice that $g(v, G)=1$ when $d \leq 2$ and that $g\left(v, T_{k}\right)=k$ for the full $k$-tree $T_{k}, k \geq 2$.

By (1.20) and Proposition A (Appendix), $s(v, n)$ may be substituted for $b(v, n)$ in (1.24) whenever $b(v, n)$ is a strictly increasing sequence, i.e., whenever $v$ lies in an infinite connected component of $G$.

Proposition 1.4. Let $G=(V, N)$ be a locally finite and connected graph, and let $v, w \in V$. Then $g(v, G)=g(w, G)$.

Proof. $G$ is connected and so let $\rho(v, w)=k \in \mathbb{N}_{0}$. By $(1.21), b(v, n)^{1 / n} \leq$ $b(w, n+k)^{1 / n}=\left(b(w, n+k)^{1 /(n+k)}\right)^{(1+k / n)}$ so that

$$
\begin{aligned}
g(v, G) & =\limsup _{n \in \mathbb{N}} b(v, n)^{1 / n} \leq \limsup _{n \in \mathbb{N}}\left(b(w, n)^{1 / n}\right)^{(1+k / n)} \\
& =\limsup _{n \in \mathbb{N}} b(w, n)^{1 / n}=g(w, G) .
\end{aligned}
$$

Similarly, $g(w, G) \leq g(v, G)$.

Definition 1.5. Let $G=(V, N)$ be a locally finite connected graph $G$. Then the growth $g(G)$ of $G$ is defined by

$$
g(G):=g(v, G)
$$

where $v$ is any vertex of $V$.

$G$ has subexponential growth (SEG) iff $g(G)=1$.

Remark 1.6. (i) The graph $G_{1}$ of Example 1 has SEG, as with $V_{0}=\left\{v_{0}\right\}$ we have $b\left(v_{0}, n\right)=\sum_{0 \leq j \leq n}\left\lfloor\frac{j+2}{2}\right\rfloor \leq(n+1)^{2}$. By Remark $1.2 G_{1}$ does not have BD.

(ii) The graph $G_{2}^{(k)}$ of Example 2 has BD, as $d\left(G_{2}^{(k)}\right)=2 k+1$ by (1.12). It does not have SEG, as by (1.18) $1<g\left(G_{2}^{(k)}\right)=1+\frac{1}{k}$.

Theorem 1. Let $G$ be a connected graph of bounded degree and of subexponential growth. Then $G$ has the period-two-property.

It is readily checked that the $\mathrm{p} 2 \mathrm{p}$ holds whenever $d(G) \leq 2$. Thus, Theorem 1 is a consequence of

Theorem 2. Let $G$ be a connected graph satisfying $3 \leq d(G)<\aleph_{0}$, and let $d \in \mathbb{N}$ be the greatest even integer satisfying $d<d(G)$. If

$$
g(G)<1+\frac{2}{d}
$$

then $G$ has the period-two-property.

Theorem 2 is proved in $\S 4$.

Corollary 1.7. Let $V$ be a nonempty subset of the $k$-dimensional Euclidean space $\mathbb{R}^{k}$; and for $u, v \in \mathbb{R}^{k}$ let $\rho_{k}(u, v)$ denote the Euclidean distance between $u$ and $v$. Let $h, r \in \mathbb{R}, 0<h, r ;$ and assume

$$
2 h<\rho_{k}(u, v) \quad(u, v \in V) .
$$

Let $G=(V, N)$, where $N \subseteq V \times V$ is defined by

$$
u N v: \Leftrightarrow 0<\rho_{k}(u, v) \leq r \quad(u, v \in V) .
$$

Then $G$ has the $p 2 p$. 
Proof. We may assume that $G$ is connected, as $G$ has the $\mathrm{p} 2 \mathrm{p}$ iff so do all its connected components. For $u \in \mathbb{R}^{k}, s \geq 0$ let $B_{k}(u, s) \subseteq \mathbb{R}^{k}$ denote the Euclidean ball centered at $u$ of radius $s$. Then for $v \in V, n \in \mathbb{N}$, the set $B(v, n) \subseteq V$ defined by (1.19) satisfies $B(v, n) \subseteq B_{k}(v, n r) \cap V$ and, in particular, $N_{v} \subseteq B_{k}(v, r)$. By $(1.26)$ we have $B_{k}(u, h) \cap V=\{u\}$ and $B_{k}(u, h) \cap B_{k}(v, h)=\varnothing$ for all $u, v \in V, u \neq v$. Hence for any $u \in \mathbb{R}^{k}$ and positive $s,\left|B_{k}(u, s) \cap V\right| \leq\left(1+\frac{s}{h}\right)^{k}$, this being the ratio of the volumes of balls of radii $s+h$ and $h$ in $\mathbb{R}^{k}$. Thus, if $v \in V, d_{G}(v)=\left|\left(B_{k}(v, r) \cap V\right) \backslash\{v\}\right| \leq$ $\left(1+\frac{r}{h}\right)^{k}$ and so $G$ satisfies $\mathrm{BD}$; similarly, for $n \in \mathbb{N}, b(v, n)=|B(v, n)| \leq$ $\left|B_{k}(v, r n) \cap V\right| \leq\left(1+\frac{n r}{h}\right)^{k}$, whence $G$ satisfies SEG. By Theorem $1 G$ has the p2p.

Let $C$ be any set with at least two elements, let $G=(V, N)$ be a locally finite connected graph, let $X={ }^{V} C$ be the set of $C$-configurations of $G$, and let $\widetilde{M}: X \rightarrow X$. Let us say that $G$ has the $\widetilde{M}-p 2 p$ if $\widetilde{M}$ has the p2p. By Remarks 1.1, 1.3, and 1.6, Examples 1 and 2 present $G_{1}$ with SEG but not BD and $G_{2}^{(k)}$ with BD but not SEG that fail to have the $\widetilde{M}$-p2p for any $\widetilde{M}$ satisfying (1.6). Theorem 1 provides that when a connected graph $G$ has BD and SEG, $G$ has the $M-\mathrm{p} 2 \mathrm{p}$ for the majority operator $M$ defined in (1.1)-which satisfies (1.6). An interesting problem we leave open is: find the extent of locally defined operators $\widetilde{M}$ satisfying (1.6) for which every connected graph with BD and SEG has the $\widetilde{M}$-p2p. Here we present just one more such example-the $k$-majority operator $M^{(k)}$, introduced in [PS], which we now precisely define.

Let $k \in \mathbb{N}, k>1$; and let $C=\mathbb{Z}_{k}=\{0,1, \ldots, k-1\}$ be the set of colors. Given a graph $G=(V, N)$, let $X={ }^{V} C$ denote the set of all $C$-configurations $x: V \rightarrow \mathbb{Z}_{k}$ of $G$. The mapping $M^{(k)}: X \rightarrow X$ is defined by putting for each $v \in V, x \in X, i \in \mathbb{Z}_{k}$ :

$$
\begin{aligned}
\left(M^{(k)} x\right)(v)=i: \Leftrightarrow \forall j \in \mathbb{Z}_{k}\left[d_{v}(x, j)\right. & \leq d_{v}(x, i) \& \\
\left(d_{v}(x, j)\right. & \left.\left.=d_{v}(x, i) \Rightarrow i \leq j\right)\right] .
\end{aligned}
$$

Thus, $\left(M^{(k)} x\right)(v)$ is the first among the colors in $\mathbb{Z}_{k}$ that $x$ applies most in $N_{v}$. as

In [PS] the $M^{(k)}-\mathrm{p} 2 \mathrm{p}$ is established for any finite graph $G$. We generalize it

Theorem 3. Let $G$ be a connected graph, with bounded degree and subexponential growth. Then $G$ has the $M^{(k)}-p 2 p$ for every $k \in \mathbb{N}$.

Theorem 3 follows from:

Theorem 4. Let $G$ be a connected graph, and let $d=d(G) \in \mathbb{N}, d>1$. If

$$
g(G)<\left(1+\frac{2}{d-1}\right)^{1 / k}
$$

then $G$ has the $M^{(k)}-p 2 p$.

Theorem 4 is proved in $\S 5$. 
We are grateful to Ron Holzman for his careful reading and critical remarks that improved an earlier version of the paper.

\section{A CRITERION FOR THE P2P}

Let $X$ be a nonempty set, and let $M: X \rightarrow X$ be an arbitrary self-mapping of $X$. Let $P$ be a partially ordered set. A mapping $b: X \times X \rightarrow P$ is called a $p 2 p$-form for $M$ in $P$ iff the following three conditions hold for all $x, y \in X$ :

$$
\begin{aligned}
& b(x, y)=b(y, x), \\
& b(x, y) \leq b(x, M x), \\
& b(x, y)=b(x, M x) \Rightarrow y=M x .
\end{aligned}
$$

We say that $M$ has the period-two-property (p2p) iff for every $x \in X$ and any positive integer $k$ :

$$
M^{k} x=x \Rightarrow M^{2} x=x .
$$

Theorem 2.0. Let $M$ be a self-mapping of a set $X$. The following are equivalent:

(a) $M$ has the $p 2 p$.

(b) There exists a partial order $P$ and a p2p form $b: X \times X \rightarrow P$ for $M$ in $P$.

Proof. (a) $\Rightarrow\left(\right.$ b): Let $P$ be the set $P\left(X^{*}\right)$ of all subsets of a set $X^{*}$ properly containing $X$ ordered by inverse inclusion:

$$
B \leq C \quad \text { iff } \quad C \subseteq B \quad\left(B, C \subseteq X^{*}\right) .
$$

Thus $X^{*}<B$ for any $B \subseteq X$.

We define $b: X \times X \rightarrow P$ as follows:

$$
\begin{aligned}
& b(x, M x)=b(M x, x):=\left\{M^{n} x: n \in \mathbb{N}_{0}\right\} \quad\left(=\left\{x, M x, M^{2} x, \ldots\right\}\right), \\
& b(x, y):=X^{*} \text { if } y \neq M x \text { and } x \neq M y .
\end{aligned}
$$

Then $b$ satisfies (2.0) and (2.1). Also, $b$ satisfies (2.2) iff (2.3) holds. Thus, if $M$ has the p2p, then $b$ is a p2p form for $M$ in $P$.

(b) $\Rightarrow(\mathrm{a})$ : Assume (b), and let $b: X \times X \rightarrow P$ be a p2p form for $M$ in $P$, where $P$ is some partial order. By $(2.0),(2.1)$

$$
b(x, M x) \leq b\left(M x, M^{2} x\right) \leq \cdots \leq b\left(M^{k-1} x, M^{k} x\right)
$$

for every positive integer $k$.

Assume that for some positive integer $k M^{k} x=x$ holds. Then by (2.0)

$$
b(x, M x) \leq b\left(M^{k-1} x, x\right)=b\left(x, M^{k-1} x\right) .
$$

As by (2.1) $b\left(x, M^{k-1} x\right) \leq b(x, M x)$, we have

$$
b(x, M x)=b\left(M x, M^{2} x\right)=\cdots=b\left(M^{k-1} x, x\right) .
$$

Hence, in particular, $b(x, M x)=b\left(M x, M^{2} x\right)$, so by equality (2.0) again, $b\left(M x, M^{2} x\right)=b(M x, x)$. But then, by (2.3), $M^{2} x=x$. Thus $M$ has the p2p, i.e., (a) holds.

Remark. The $\mathrm{p} 2 \mathrm{p}$ for a variety of discrete time finite dynamical systems is established often using a mapping $E$ from the configuration space $X$ of the system into the real numbers, whose time-behaviour is monotone (see, e.g., 
[GFP], [GM], [GOd], [OR]). A p2p form $b$ for $M$ in $P$ yields such a function by setting $E(x):=b(x, M x)$.

\section{A SUFFICIENT CONDITION FOR P2P IN SYMMETRIC NETWORKS}

In this section we utilize the criterion for $p 2 p$ obtained in $\S 2$ to obtain useful sufficient conditions for locally defined operators on configuration spaces over symmetric networks to have the $\mathrm{p} 2 \mathrm{p}$.

By a network we shall mean a pair $G=(V, N)$, where $V$ is a nonempty set (of vertices or nodes), and $N: V \times V \rightarrow \mathbb{R}$ is any $V \times V$ real matrix. The network is called symmetric if

$$
N(u, v)=N(v, u) \quad(u, v \in V) .
$$

A graph $G$ as defined in $\S 1$ is a special case of a symmetric network, if we interpret its neighbourhood relation as a characteristic function, namely, the function $N: V \times V \rightarrow\{0,1\}$ satisfying $N(u, v)=1$ iff $u$ and $v$ are neighbours in $G$.

Let $G=(V, N)$ be an arbitrary network, and let $C$ be a nonempty set. The set $X:={ }^{V} C$ is called again the set of $C$-configurations or $C$-colouring of $G$. Given a system $\left(M_{v}\right)_{v \in V}$ of functions $M_{v}: X \rightarrow C$ (referred to as local operators), a unique operator $M: X \rightarrow X$ is defined by

$$
M x(v):=M_{v} x \quad(x \in X, v \in V) .
$$

Then we say that $M$ is locally defined by the system of local operators $\left(M_{v}\right)_{v \in V}$. A useful sufficient condition for a locally defined $M: X \rightarrow X$ to have the p2p is given in the next theorem.

Theorem 3.0. Let $V, C$ be nonempty sets, and let $X={ }^{V} C$. Let $b: X \times X \rightarrow \mathbb{R}$; and for each $v \in V$ let $b_{v}: X \times C \rightarrow \mathbb{R}, M_{v}: X \rightarrow C$ be given so that:

(b0) $b(x, y)=b(y, x) \quad(x, y \in X)$.

(b1) $b(x, y)=\sum_{v \in V} b_{v}(x, y(v))$, where the sum is absolutely convergent $(x, y \in X)$.

(b2) $b_{v}(x, c) \leq b_{v}\left(x, M_{v} x\right) \quad(v \in V, x \in X, c \in C)$.

(b3) $b_{v}(x, c)=b_{v}\left(x, M_{v} x\right) \Rightarrow c=M_{v} x \quad(v \in V, x \in X, c \in C)$.

Let $M: X \rightarrow X$ be defined by

$$
M x(v):=M_{v} x \quad(x \in X, v \in V) .
$$

Then $M$ has the $p 2 p$.

Proof. By Theorem 2.0 it is enough to show that $b$ is a p2p form for $M$ in $\mathbb{R}$, i.e., $b$ satisfies (2.0), (2.1), and (2.2).

Indeed (2.0) holds by (b0).

Let $x, y \in X$. By (b1) and (3.1) we have

$$
b(x, M x)-b(x, y)=\sum_{v \in V}\left(b_{v}\left(x, M_{v} x\right)-b_{v}(x, y(v))\right) \quad(x, y \in X)
$$

and the sum on the right is absolutely convergent.

By (b2) $b_{v}\left(x, M_{v} x\right)-b_{v}(x, y(v)) \geq 0$ for all $v \in V$, so that by (3.2) $b(x, y) \leq b(x, M x)$, i.e., (2.1) holds.

Assume $b(x, y)=b(x, M x)$. Then the sum on the right vanishes in (3.2). As all its terms are nonnegative, we have $b_{v}(x, y(v))=b_{v}\left(x, M_{v} x\right)$ for all 
$v \in V$. Hence, by (b3) and (3.1), $y(v)=M_{v} x=M x(v)$ for all $v \in V$, i.e., $y=M x$. Thus, also (2.2) holds.

In practice, special instances of Theorem 3.0 with some more details on the structure of the function $b$ are useful. We first formulate one that is useful in handling the case $|C|=2$ and $M$ the majority operator, defined in (1.1).

Theorem 3.1. Let $G=(V, B)$ be a network, $C \subseteq \mathbb{R}$, and $X={ }^{V} C$. For each $v \in V$ let $M_{v}: X \rightarrow C$ be given, and let $M: X \rightarrow X$ be defined by

$$
M x(v):=M_{v}(x) \quad(x \in X, v \in V) .
$$

Assume that $B: V \times V \rightarrow \mathbb{R}$ satisfies

(B0) $B(u, v)=B(v, u) \quad(u, v \in V)$.

(B1) $\sum_{(u, v) \in V \times V}|x(u)||B(u, v)||y(v)|<\infty \quad(x, y \in X)$.

Further let

$$
\begin{aligned}
& b(x, y)=\sum_{(u, v) \in V \times V} x(u) B(u, v) y(v) \quad(x, y \in X), \\
& b_{v}(x, c)=c \sum_{u \in V} x(u) B(u, v) \quad(v \in V, x \in X, c \in C) .
\end{aligned}
$$

If

(b2) $b_{v}(x, c) \leq b_{v}\left(x, M_{v} x\right) \quad(v \in V, x \in X, c \in C)$.

(b3) $b_{v}(x, c)=b_{v}\left(x, M_{v} x\right) \Rightarrow c=M_{v} x \quad(v \in V, x \in X, c \in C)$. Then $M$ has the $p 2 p$.

Notice that if $C \subseteq \mathbb{R}$ is bounded-in particular, if $C$ is finite-then (B1) is a consequence of

(B'1) $\sum_{(u, v) \in V \times V}|B(u, v)|<\infty$.

(In fact, (B1) is equivalent to $\left(\mathrm{B}^{\prime} 1\right)$ when $C$ has a nonzero number.) The proof of Theorem 2 in $\S 4$ makes use of Theorem 3.1. Now we formulate a slight generalization used to prove Theorem 4 in $\S 5$.

Theorem 3.2. Let $G=(V, B)$ be a network, $C \subseteq \mathbb{R}$, and $X={ }^{V} C$. For each $v \in V$ let $M_{v}: X \rightarrow C$ be given and let $M: X \rightarrow X$ be defined by

$$
M x(v):=M_{v} x \quad(x \in X, v \in V) .
$$

Let $B: V \times V \rightarrow \mathbb{R}, K: C \times C \rightarrow \mathbb{R}$ satisfy

(BK0) $B(u, v)=B(v, u), K\left(c^{\prime}, c^{\prime \prime}\right)=K\left(c^{\prime \prime}, c^{\prime}\right) \quad\left(u, v \in V ; c^{\prime}, c^{\prime \prime} \in C\right)$.

(BK1) $\sum_{(u, v) \in V \times V}|x(u)||B(u, v)||K(x(u), y(v))||y(v)|<\infty \quad(x, y \in X)$.

Further let

$$
\begin{aligned}
b(x, y) & :=\sum_{(u, v) \in V \times V} x(u) B(u, v) K(x(u), y(v)) y(v) \quad(x, y \in X), \\
b_{v}(x, c) & :=c \sum_{u \in V} x(u) B(u, v) K(x(u), c) \quad(v \in V, x \in X, c \in C) .
\end{aligned}
$$

If

(b2) $b_{v}(x, c) \leq b_{v}\left(x, M_{v} x\right) .(v \in V, x \in X, c \in C)$.

(b3) $b_{v}(x, c)=b_{v}\left(x, M_{v} x\right) \Rightarrow c=M_{v} x \quad(v \in V, x \in X, c \in C)$.

Then $M$ has the $p 2 p$. 
Again, if $C \subseteq \mathbb{R}$ is bounded and $K$ is a bounded function, then (BK1) is a consequence of $\left(B^{\prime} 1\right)$.

\section{Proof of Theorem 2}

Let $G^{\prime}=\left(V, N^{\prime}\right)$ be a connected graph, where $N^{\prime}$ is a symmetric irreflexive relation on $V$, i.e., satisfies (N1) and (N2) (see $\S 1)$. Let $3 \leq d\left(G^{\prime}\right) \in \mathbb{N}$ and $d \in$ $\mathbb{N}$ be the greatest even integer satisfying $d<d\left(G^{\prime}\right)$, and assume $g\left(G^{\prime}\right)<1+\frac{2}{d}$. Let $C=\{-1,1\}$, and let $M$ denote the majority operator on $X:={ }^{V} C$ as defined in (1.1). We shall define a network $G=(V, B)$ so that the assumptions of Theorem 3.1 hold, whence we conclude that $M$ has the p2p. First some preliminaries.

Fix a positive $q \in \mathbb{R}$ satisfying

$$
\frac{d}{d+2}<q^{2}<\frac{1}{g\left(G^{\prime}\right)}
$$

As $1 \leq g\left(G^{\prime}\right), q$ satisfies $0<q<1$.

Fix $v_{0} \in V$, and for any $v \in V$ put

$$
n(v):=\rho\left(v_{0}, v\right)
$$

where $\rho(u, v)$ is the distance in $G^{\prime}$ between $u$ and $v$. Further let $S_{n}:=$ $S\left(v_{0}, n\right)$ be the sphere of radius $n$ centered at $v_{0}$ (see (1.19)) so that $n(v)=n$ iff $v \in S_{n}$; and let $s_{n}:=\left|S_{n}\right|$.

Define a positive number $q_{v}$ for $v \in V$ by

$$
q_{v}:=q^{n(v)} \text {. }
$$

First we show that

$$
\sum_{v \in V} q_{v}^{2}=\sum_{n \in \mathbb{N}_{0}} s_{n} q^{2 n}<\infty
$$

As $V=\bigcup_{n \in \mathrm{N}_{0}} S_{n}$ is a disjoint union, the equality in (4.3) holds, and we only need to prove the inequality, i.e. the convergence of the series of nonnegative terms $\sum_{n \in \mathrm{N}_{0}} s_{n} q^{2 n}$. Indeed, $\left(s_{n} q^{2 n}\right)^{1 / n}=q^{2} s_{n}^{1 / n}$, hence by (4.0), (1.24), and Definition 1.5

$$
\limsup _{n \in \mathrm{N}}\left(s_{n} q^{2 n}\right)^{1 / n}=q^{2} \limsup _{n \in \mathbb{N}} s_{n}^{1 / n} \leq q^{2} \limsup _{n \in \mathbb{N}}\left(\sum_{0 \leq i \leq n} s_{i}\right)^{1 / n}=q^{2} g\left(G^{\prime}\right)<1 .
$$

The inequality in (4.3) follows.

We use $d_{v}$ as a brief notation for the degree of $v \in V$ in $G^{\prime}$. Thus:

$$
N_{v}:=\left\{u \in V: u N^{\prime} v\right\}, \quad d_{v}:=\left|N_{v}\right| .
$$

By the choice of $d$ we have

$$
\begin{aligned}
& d_{v} \leq d+1 \quad \text { if } d_{v} \text { is odd } \\
& d_{v} \leq d+2 \quad \text { if } d_{v} \text { is even }
\end{aligned} \quad(v \in V) .
$$

Let $v \in V$ and let $n=n(v)$. Then obviously $N_{v} \subseteq S_{n-1} \cup S_{n} \cup S_{n+1}$ (where by definition $\left.S_{-1}=\varnothing\right)$, so $u \in N_{v}$ implies $n(u) \in\{n-1, n, n+1\}$. 
Define $m_{v}: N_{v} \rightarrow \mathbb{R}$ by $m_{v} u:=q_{v}^{-1} q_{u}$ so that $m_{v} u=q^{n(u)-n} \in\left\{q^{-1}, 1, q\right\}$ for any $u \in N_{v}$.

For $A \subseteq N_{v}$ put

$$
m_{v} A:=\sum_{u \in A} m_{v} u, \quad w_{v} A:=m_{v} A-m_{v}\left(N_{v}-A\right) .
$$

$m_{v}$ and $w_{v}$ respect cardinality in $N_{v}$; that is,

$$
|A|<|B| \Rightarrow m_{v} A<m_{v} B \& w_{v} A<w_{v} B \quad\left(A, B \subseteq N_{v}\right) .
$$

We prove (4.6). First let $|A|=j,|B|=j+1$. Then

$$
m_{v} A \leq j q^{-1}, \quad(j+1) q \leq m_{v} B .
$$

First assume $j<d_{v} / 2$. In this case $m_{v} A<m_{v} B$ follows from $j q^{-1}<$ $(j+1) q$, i.e., from $\frac{j}{j+1}=1-\frac{1}{j+1}<q^{2}$. Indeed, by (4.0),

$$
1-\frac{1}{d / 2+1}<q^{2}
$$

and $\frac{d}{2}$ is the largest integer smaller than $\frac{d\left(G^{\prime}\right)}{2}$, so that $j<d_{v} / 2$ implies $j \leq \frac{d}{2}$, whence $1-\frac{1}{j+1} \leq 1-\frac{1}{d / 2+1}<q^{2}$.

Now assume that $j \geq d_{v} / 2$. Then $|B|=j+1>d_{v} / 2$ and so $B^{c}:=$ $N_{v}-B$ satisfies $\left|B^{c}\right|=d_{v}-(j+1)<d_{v} / 2$. As $A^{c}:=N_{v}-A$ satisfies $\left|A^{c}\right|=d_{v}-j=\left|B^{c}\right|+1$ we have by the previous case $m_{v} B^{c}<m_{v} A^{c}$. By $m_{v} N_{v}=m_{v} B+m_{v} B^{c}=m_{v} A+m_{v} A^{c}, m_{v} A<m_{v} B$ follows.

Thus we see that $|A|<|B|$ implies $m_{v} A<m_{v} B$ for all $A, B \subseteq N_{v} . w_{v} A<$ $w_{v} B$ readily follows, as $|A|<|B| \Rightarrow\left|B^{c}\right|<\left|A^{c}\right|$ and so also $w_{v} A=m_{v} A-$ $m_{v} A^{c}<m_{v} B-m_{v} B^{c}=w_{v} B$. (4.6) is established.

We also obviously have

$$
w_{v}\left(N_{v}-A\right)=-w_{v} A \quad\left(A \subseteq N_{v}\right) .
$$

Proposition 4.0. Let $v \in V$. Then:

(1) $A \subseteq N_{v},|A|<\frac{1}{2} d_{v} \Rightarrow w_{v} A<0<w_{v}\left(N_{v}-A\right)$.

(2) There is a positive number $\delta_{v}>0$ such that if $A, B, C \subseteq N_{v}$ satisfy $|B|<|A|=\frac{1}{2} d_{v}<|C|$ then

$$
w_{v} B<-\delta_{v}<w_{v} A<\delta_{v}<w_{v} C .
$$

Proof. (1) follows from (4.6) and (4.7).

(2) is of relevance only when $d_{v}$ is even and follows similarly. (Notice that $|A|=\frac{1}{2} d_{v}$ implies $\left|N_{v}-A\right|=\frac{1}{2} d_{v}$, so by (4.7) the set of numbers $\left\{w_{v} A:|A|=\right.$ $\left.\frac{1}{2} d_{v}\right\}$ is symmetric around zero.)

Let us define $N: V \times V \rightarrow \mathbb{R}$ by

$$
N(u, v):= \begin{cases}1, & u N^{\prime} v \\ \delta_{v}, & u=v \text { and } d_{v} \text { is even } \\ 0, & \text { otherwise. }\end{cases}
$$

Thus, $N$ is symmetric and differs from the characteristic function of the symmetric relation $N^{\prime}$ yielding the graph $G^{\prime}$ by introducing the diagonal positive entries $\delta_{v}$ satisfying (4.8) for those $v \in V$ where $d_{v}$ is even. 
Define the network $G=(V, B)$ by setting

$$
B(u, v):=q_{u} N(u, v) q_{v} \quad(u, v \in V) .
$$

Then $B$ is symmetric, so (B0) holds.

As $C=\{-1,1\}$, (B1) follows once we establish (B'1). Notice first that for each $v \in V$ we have $\sum_{u \in N_{v}} q_{u} \leq q_{v} q^{-1} d_{v} \leq q_{v} q^{-1}(d+2)$. Also, whenever $d_{v}$ is even, $\delta_{v}<w_{v} N_{v}=m_{v} N_{v} \leq q^{-1} d_{v} \leq q^{-1}(d+2)$. Hence by (4.3),

$$
\begin{aligned}
\sum_{(u, v) \in V \times V}|B(u, v)| & <\sum_{v \in V}\left(q^{-1}(d+2) q_{v}^{2}+q_{v} \sum_{u \in N_{v}} q_{u}\right) \\
& \leq 2 q^{-1}(d+2) \sum_{v \in V} q_{v}^{2}<\infty .
\end{aligned}
$$

Thus, (B'1) holds.

As in $\S 1$ we put for $x: V \rightarrow C, v \in V, c \in C$,

$$
N_{v}(x, c):=\left\{u \in N_{v}: x(u)=c\right\}, \quad d_{v}(x, c):=\left|N_{v}(x, c)\right|
$$

and define $M_{v}: X \rightarrow C$ by

$$
M_{v} x= \begin{cases}c & \text { if } \frac{1}{2} d_{v}<d_{v}(x, c) \\ x(v) & \text { if } d_{v}(x, 1)=d_{v}(x,-1)\end{cases}
$$

Thus, $M: X \rightarrow X$ defined by (3.1) is the majority operator.

For $x, y \in X, v \in V, c \in C$ defined $b(x, y) \in \mathbb{R}$ and $b_{v}(x, c) \in \mathbb{R}$ by (3.3). Then we have

$$
\begin{cases}b_{v}(x, c)=q_{v}^{2} w_{v} N_{v}(x, c) & \text { if } d_{v} \text { is odd } \\ b_{v}(x, c)=q_{v}^{2}\left(w_{v} N_{v}(x, c)+c x(v) \delta_{v}\right) & \text { if } d_{v} \text { is even. }\end{cases}
$$

Indeed, by (3.3), (4.4), (4.9), (4.10),

$$
\begin{aligned}
b_{v}(x, c) & =c \sum_{u \in V} x(u) B(u, v)=q_{v}\left(q_{v} c x(v) N(v, v)+\sum_{u \in N_{v}}(x(u) c) q_{u}\right) \\
& =q_{v}^{2}\left(c x(v) N(v, v)+m_{v} N_{v}(x, c)-m_{v} N_{v}(x,-c)\right) \\
& =q_{v}^{2}\left(c x(v) N(v, v)+w_{v} N_{v}(x, c)\right) .
\end{aligned}
$$

Hence (4.13) follows from (4.9).

(b2) and (b3) follow from

$$
b_{v}\left(x,-M_{v} x\right)<0<b_{v}\left(x, M_{v} x\right) \quad(v \in V, x \in X) .
$$

To prove (4.14), put $c=M_{v} x, A=N_{v}(x, c)$. Then by (4.12), $\left|N_{v}-A\right| \leq$ $\frac{1}{2} d_{v} \leq|A|$.

First assume that $d_{v}$ is odd. Then we have $\left|N_{v}-A\right|<\frac{1}{2} d_{v} \leq|A|$. Hence by Proposition 4.0(1) and $N_{v}-A=N_{v}(x,-c)$ we have

$$
w_{v} N_{v}(x,-c)<0<w_{v} N_{v}(x, c),
$$

whence (4.14) follows by (4.13).

Next assume that $d_{v}$ is even, and let $w:=w_{v} N_{v}(x, c)$, so by (4.13) and (4.7),

$$
b_{v}(x, c)=q_{v}^{2}\left(w+c x(v) \delta_{v}\right), \quad b_{v}(x,-c)=-b_{v}(x, c) .
$$


Thus, (4.14) follows once we show $w+c x(v) \delta_{v}>0$. Indeed, if $\left|N_{v}(x, c)\right|>$ $\frac{1}{2} d_{v}$, then by (4.8) $w=w_{v} N_{v}(x, c)>\delta_{v}>0$ and so $w+c x(v) \delta_{v} \geq w-\delta_{v}>0$; and if $\left|N_{v}(x, c)\right|=\frac{1}{2} d_{v}$, then $c=x(v)$ by (4.12), so $w+c x(v) \delta_{v}=w+\delta_{v}$ and again by (4.8) $w+\delta_{v}>0$.

\section{Proof of Theorem 4}

Let $k \in \mathbb{N}, k>1$, and let $G=(V, N)$ be a connected graph satisfying $d:=d(G) \in \mathbb{N}, d>1$, and

$$
g(G)<\left(1+\frac{2}{d-1}\right)^{1 / k}
$$

We consider $G$ as a network (so that $N: V \times V \rightarrow\{0,1\}$ is a symmetric function) and apply Theorem 3.2 to show that $G$ has the $M^{(k)}$-p2p (see (1.27)).

By $(1.28) g(G)<\left(\frac{d+1}{d-1}\right)^{2 /(2(k-1)+2)}$. Choose $\alpha>2$ so that

$$
g(G)<\left(\frac{d+1}{d-1}\right)^{2 /(\alpha(k-1)+2)}
$$

still holds, and fix $0<q<1$ satisfying

$$
\left(\frac{d-1}{d+1}\right)^{\frac{2}{\alpha(k-1)+2}}<q^{2}<\frac{1}{g(G)} .
$$

The set of $k$ colours $C \subseteq \mathbb{R}$ is defined by

$$
C:=\left\{q^{\alpha i / 2}: i \in \mathbb{Z}_{k}\right\} \text {. }
$$

so $i \mapsto q^{\alpha i / 2}$ is an order-reversing mapping of $\mathbb{Z}_{k}$ onto $C$ (as $0<q<1$ ). We put further $X:={ }^{V} C$, and for $v \in V, x \in X$, and $c \in C$ put $N_{v}:=\{u \in$ $V: N(u, v)=1\}, d_{v}:=\left|N_{v}\right|$ and define $N_{v}(x, c), d_{v}(x, c)$ by (4.11).

Let us put $c_{i}:=q^{\alpha i / 2}$ for $i \in \mathbb{Z}_{k}$ so that $C=\left\{c_{i}: i \in \mathbb{Z}_{k}\right\}$, and guided by (1.27) let us define the local operator $\widetilde{M}_{v}: X \rightarrow C$ for each $v \in V$ by

$$
\begin{aligned}
\widetilde{M}_{v}(x)=c_{i}: \Leftrightarrow \forall j \in \mathbb{Z}_{k}\left[d_{v}\left(x, c_{j}\right)\right. & \leq d_{v}\left(x, c_{i}\right) \& \\
\left(d_{v}\left(x, c_{j}\right)\right. & \left.\left.=d_{v}\left(x, c_{i}\right) \Rightarrow i \leq j\right)\right] .
\end{aligned}
$$

Finally, $\widetilde{M}: X \rightarrow X$ is defined by

$$
\widetilde{M} x(v):=\widetilde{M}_{v}(x) .
$$

To establish Theorem 4 we need to show that $\widetilde{M}$ has the p2p. By Theorem 3.2 it suffices to define $B: V \times V \rightarrow \mathbb{R}$ and $K: C \times C \rightarrow \mathbb{R}$ so that (BK0), (BK1) hold and so that $b, b_{v}$ defined in (3.4) satisfy (b2) and (b3).

Choose $v_{0} \in V$, and define

$$
n(v):=\rho\left(v_{0}, v\right), \quad q_{v}:=q^{n(v)} \quad(v \in V) .
$$

Define $B: V \times V \rightarrow \mathbb{R}, K: C \times C \rightarrow\{0,1\}$ by

$$
B(u, v):=q_{u} N(u, v) q_{v}, \quad K\left(c, c^{\prime}\right)=\delta\left(c, c^{\prime}\right) \quad\left(u, v \in V ; c, c^{\prime} \in C\right)
$$

where $\delta\left(c, c^{\prime}\right)=1$ if $c=c^{\prime}, \delta\left(c, c^{\prime}\right)=0$ otherwise.

By (5.5), (BK0) holds (as $N$ is symmetric). 
As $C$ is finite, $K\left(c, c^{\prime}\right) \in\{0,1\},(\mathrm{BK} 1)$ follows from $\left(\mathrm{B}^{\prime} 1\right)$. By (5.0) $q^{2} g(G)<1$, so by (5.4), Definition 1.5, and (5.0) $\sum_{v \in V} q_{v}^{2}<\infty$ (see (4.3)). Hence, we have again $\sum_{(u, v) \in V \times V}|B(u, v)|<\infty$. (See (4.10) and the following lines, §4.) Thus ( $\left.\mathbf{B}^{\prime} 1\right)$ and, hence, (BK1) hold.

Substituting (5.5) into (3.4) we obtain

$$
\begin{aligned}
& b(x, y)=\sum_{(u, v) \in V \times V} x(u) q_{u} N(u, v) \delta(x(u), y(v)) q_{v} y(v) \quad(x, y \in X), \\
& b_{v}(x, c)=c q_{v} \sum_{u \in V} x(u) q_{u} N(u, v) \delta(x(u), c) \quad(v \in V, x \in X, c \in C) .
\end{aligned}
$$

We notice that with $N_{v}(x, c), d_{v}(x, c)$ defined in (4.11) (or (1.0)) we have $N(u, v) \delta(x(u), c)=1$ or 0 accordingly as $u \in N_{v}(x, c)$ or $u \notin N_{v}(x, c)$. Thus, for any $v \in V, x \in X, c \in C$,

$$
b_{v}(x, c)=c^{2} q_{v} \sum_{u \in N_{v}(x, c)} q_{u}
$$

Also, for $u \in N_{v}$

Hence

$$
q q_{v} \leq q_{u} \leq q^{-1} q_{v}
$$

$$
c^{2} q q_{v}^{2} d_{v}(x, c) \leq b_{v}(x, c) \leq c^{2} q^{-1} q_{v}^{2} d_{v}(x, c) .
$$

Let us put $n:=n(v)$ so that $q_{v}=q^{n}$, and by (5.1) we may put $c^{2}=q^{\alpha i}$ for some integer $0 \leq i<k$. Then (5.6) gives

$$
q^{2 n+\alpha i+1} d_{v}\left(x, q^{\alpha i / 2}\right) \leq b_{v}\left(x, q^{\alpha i / 2}\right) \leq q^{2 n+\alpha i-1} d_{v}\left(x, q^{\alpha i / 2}\right) .
$$

Claim 1. Let $c, c^{\prime} \in C, c<c^{\prime}$; and let $0<m=d_{v}(x, c)=d_{v}\left(x, c^{\prime}\right)$. Then $b_{v}(x, c)<b_{v}\left(x, c^{\prime}\right)$.

Indeed we may assume $c=q^{\alpha i / 2}, c^{\prime}=q^{\alpha j / 2}$ where $0 \leq j<i<k$. Then by (5.7)

$$
b_{v}(x, c) \leq q^{2 n+\alpha i-1} m<q^{2 n+\alpha j+1} m \leq b_{v}\left(x, c^{\prime}\right)
$$

as required in Claim 1.

Claim 2. Let $c, c^{\prime} \in C$ and let $0 \leq m=d_{v}(x, c)<m^{\prime}=d_{v}\left(x, c^{\prime}\right)$. Then $b_{v}(x, c)<b_{v}\left(x, c^{\prime}\right)$.

Indeed, by $m+1 \leq m^{\prime}$ and (5.7) we have for $c=q^{\alpha i / 2}, c^{\prime}=q^{\alpha j / 2}$ :

$$
\begin{aligned}
b_{v}(x, c) \leq q^{2 n+\alpha i-1} m & \leq q^{2 n-1} m, \\
q^{2 n+\alpha(k-1)+1}(m+1) & \leq q^{2 n+\alpha(k-1)+1} m^{\prime} \leq q^{2 n+\alpha j+1} m^{\prime} \leq b_{v}\left(x, c^{\prime}\right) .
\end{aligned}
$$

Now $2 m+1 \leq m+m^{\prime} \leq d_{v} \leq d$, so $m \leq d-(m+1)$. Hence:

$$
m(d+1)=m d+m \leq m d+d-(m+1)=(m+1)(d-1) .
$$

Thus, by (5.0)

$$
\frac{m}{m+1} \leq \frac{d-1}{d+1}<q^{\alpha(k-1)+2} .
$$

Multiplying by $(m+1) q^{2 n-1}$ we obtain

$$
q^{2 n-1} m<q^{2 n+\alpha(k-1)+1}(m+1)
$$

combining (5.8), (5.9), and (5.10) we conclude $b_{v}(x, c)<b_{v}\left(x, c^{\prime}\right)$, as Claim 2 asserts.

We conclude from Claims 1 and 2: 
Corollary. $b_{v}(x, c)<b_{v}\left(x, c^{\prime}\right)$ iff

$$
d_{v}(x, c)<d_{v}\left(x, c^{\prime}\right) \quad \text { or } \quad\left(d_{v}(x, c)=d_{v}\left(x, c^{\prime}\right) \& c<c^{\prime}\right) .
$$

Comparing the corollary with (5.2) we conclude

(b2) $b_{v}(x, c) \leq b_{v}\left(x, \widetilde{M}_{v} x\right) \quad(v \in V, x \in X, c \in C)$,

(b3) $b_{v}(x, c)=b_{v}\left(x, \widetilde{M}_{v} x\right) \Rightarrow c=\widetilde{M}_{v} x \quad(v \in V, x \in X, c \in C)$.

As all assumptions of Theorem 3.2 hold, $\widetilde{M}$ has the p2p.

Theorem 4 (hence Theorem 3) is proved.

\section{APPENDIX}

Proposition A. Let $a_{n}>0$ for $n \in \mathbb{N}, \tilde{a}_{n}=a_{n}^{1 / n}, b_{n}=\sum_{1 \leq i \leq n} a_{i}, \tilde{b}_{n}=b_{n}^{1 / n}$; and let $0 \leq \tilde{a}, \tilde{b} \leq \infty$ satisfy $\tilde{a}=\lim \sup _{n \in \mathbb{N}} \tilde{a}_{n}, \tilde{b}=\lim \sup _{n \in \mathbb{N}} \tilde{b}_{n}$. Then $\tilde{b}=\max (1, \tilde{a})$. In particular, if $a_{n} \geq 1$ for all $n$, then $\tilde{b}=\tilde{a}$.

Proof. As $\tilde{a}_{n} \leq \tilde{b}_{n}$, we have $\tilde{a} \leq \tilde{b}$. Also, as $\tilde{b}_{n}>a_{1}^{1 / n}, a_{1}>0$, we have $1 \leq \liminf _{n \in \mathbb{N}} \tilde{b}_{n} \leq \tilde{b}$. Thus, $\max (\tilde{a}, 1) \leq \tilde{b}$. For the reverse inequality we prove:

Claim. Let $K$ satisfy $1<K$ and $\tilde{a}_{n}<K$ for all large enough $n \in \mathbb{N}$. Then $\tilde{b} \leq K$.

Proof of Claim. Assume that $1<N \in \mathbb{N}$ satisfies $\tilde{a}_{n}<K$ for all $n \geq N$. As $a_{j}<K^{j}$ for $j \geq N$ we have for all $n \geq N$

$$
b_{n}=b_{N-1}+\sum_{N \leq j \leq n} a_{j}<b_{N-1}+\sum_{N \leq j \leq n} K^{j}
$$

Thus, if $L=\max \left(1, b_{N-1}\right)$, then

$$
b_{n}<L \sum_{0 \leq j \leq n} K^{j}=\frac{L}{K-1}\left(K^{n+1}-1\right)<\frac{L K}{K-1} \cdot K^{n}
$$

and so

$$
\tilde{b}_{n}=b_{n}^{1 / n}<\left(\frac{L K}{K-1}\right)^{1 / n} K
$$

whence $\tilde{b} \leq K$.

$\tilde{b} \leq \max (1, \tilde{a})$ follows from the claim and completes the proof of Proposition A.

\section{REFERENCES}

[GFP] E. Goles, F. Fogelman-Soulie, and D. Pellegrin, Decreasing energy functions as a tool for studying threshold networks, Discrete Appl. Math. 12 (1985), 261-277.

[GM] E. Goles and S. Martinez, Neural and automata networks, Kluwer Academic, Dordrecht, 1990.

[GO] E. Goles and J. Olivos, Periodic behaviour of generalized threshold functions, Discrete Math. 30 (1980), 187-189.

[GOd] E. Goles and A. Odlyzko, Decreasing energy functions and the lengths of transients for some cellular automata, Complex Systems 2 (1988), 501-507.

[M1] G. Moran, The r-majority-vote action on 0-1 sequences, Discrete Math. 132 (1994), 145-174. 
[M2] _ Parametrization for stationary patterns of the r-majority operators on 0-1 sequences, Discrete Math. 132 (1994), 175-195.

[OR] A. M. Odlyzko and D. J. Randal, On the periods of some graph transformations, Complex Systems 1 (1987), 203-210.

[PS] S. Poljak and M. Sura, On periodical behaviour in societies with symmetric influences, Combinatorica 3 (1983), 119-121.

[PT] S. Poljak and D. Turzik, On an application of convexity to discrete systems, Discrete Appl. Math. 13 (1986), 27-32.

Department of Mathematics and Computer SCIEnCe, University of Haifa, Haifa 31905 , ISRAEL

E-mail address: rsma309@uvm.haifa.ac.il 